\title{
Computational Insights into the Interactions between Calmodulin and the c/nSH2 Domains of p85 $\alpha$ Regulatory Subunit of PI3K $\alpha$ : Implication for PI3K $\alpha$ Activation by Calmodulin
}

\author{
Duan Ni (B), Dingyu Liu, Jian Zhang and Shaoyong Lu* \\ Department of Pathophysiology, Key Laboratory of Cell Differentiation and Apoptosis of Chinese Ministry of \\ Education, School of Medicine, Shanghai Jiao Tong University, Shanghai 200025, China; \\ niduan11@sjtu.edu.cn (D.N.); sjdldy@sjtu.edu.cn (D.L.); methane02@163.com (J.Z.) \\ * Correspondence: lushaoyong@yeah.net; Tel.: +86-21-6384-6590-776-922; Fax: +86-21-6415-4900
}

Received: 1 December 2017; Accepted: 26 December 2017; Published: 4 January 2018

\begin{abstract}
Calmodulin (CaM) and phosphatidylinositide-3 kinase (PI3K $\alpha$ ) are well known for their multiple roles in a series of intracellular signaling pathways and in the progression of several human cancers. Crosstalk between CaM and PI3K $\alpha$ has been an area of intensive research. Recent experiments have shown that in adenocarcinoma, K-Ras4B is involved in the CaM-PI3K $\alpha$ crosstalk. Based on experimental results, we have recently put forward a hypothesis that the coordination of CaM and PI3K $\alpha$ with K-Ras4B forms a CaM-PI3K $\alpha$-K-Ras4B ternary complex, which leads to the formation of pancreatic ductal adenocarcinoma. However, the mechanism for the CaM-PI3K $\alpha$ crosstalk is unresolved. Based on molecular modeling and molecular dynamics simulations, here we explored the potential interactions between $\mathrm{CaM}$ and the $\mathrm{c} / \mathrm{nSH} 2$ domains of $\mathrm{p} 85 \alpha$ subunit of PI3K $\alpha$. We demonstrated that $\mathrm{CaM}$ can interact with the $\mathrm{c} / \mathrm{nSH} 2$ domains and the interaction details were unraveled. Moreover, the possible modes for the $\mathrm{CaM}-\mathrm{cSH} 2$ and $\mathrm{CaM}-\mathrm{nSH} 2$ interactions were uncovered and we used them to construct a complete CaM-PI3K $\alpha$ complex model. The structural model of CaM-PI3K $\alpha$ interaction not only offers a support for our previous ternary complex hypothesis, but also is useful for drug design targeted at CaM-PI3K $\alpha$ protein-protein interactions.
\end{abstract}

Keywords: calmodulin; PI3K $\alpha$; K-Ras4B; molecular dynamics simulations; molecular modeling; adenocarcinoma; protein-protein interactions

\section{Introduction}

K-Ras4B is situated at the crossroad of several cellular signaling pathways, and it is involved in cell growth and proliferation [1-6]. Mutations of K-Ras4B frequently occur in various kinds of cancers [3,7-11], and one of the most deadly of them is pancreatic ductal adenocarcinoma (PDAC) [9,12-14]. PDAC is known for its poor prognosis and high mortality and few patients can survive for 5 years after operations [15]. Mutation rate of K-Ras4B in PDAC can be as high as $95 \%[5,16,17]$ and accumulating evidence suggests that both calmodulin (CaM) and phosphatidylinositide-3 kinase (PI3K $\alpha$ ) play key roles in the K-Ras4B-driven adenocarcinoma [18-21].

$\mathrm{CaM}$ is a calcium-binding protein [22-24] that consists of a pair of symmetric N-lobe and $\mathrm{C}$-lobe and the connecting linker [25]. Since the linker is relatively flexible, CaM can adopt two conformational topologies, the extended and the collapsed shapes. In response to calcium binding, CaM can interact with different targets [26] and it is thus involved in several intracellular signaling pathways [22-24,27-30]. One of the major targets of CaM is K-Ras4B [18-20,27]. In adenocarcinoma, GTP-bound activated K-Ras4B is specifically regulated by CaM $[19,20,24]$, and such a finding is 
supported by the clinical observation that higher calcium concentration in serum can lead to the poor prognosis of the K-Ras4B-driven PDAC [23]. Thus, it is widely accepted that CaM can modulate the progression of K-Ras4B-driven cancer and one potential downstream target is the K-Ras4B-PI3K-Akt pathway $[19,21,24,28]$.

PI3K $\alpha$ is a downstream target of K-Ras4B [31,32] and PI3K $\alpha$-Akt signaling pathway is one of the key components in the K-Ras4B-driven tumorigenesis [21,32-34]. PI3K $\alpha$ is a lipid kinase composed of p $110 \alpha$ catalytic and p85 $\alpha$ regulatory domains [35-38]. Upon activation by the phosphorylated epidermal growth factor receptor ( $\mathrm{pEGFR}$ ) and K-Ras4B, PI3K $\alpha$ catalyzes the synthesis of the second messenger phosphatidylinositol-3,4,5-triphosphate $\left(\mathrm{PIP}_{3}\right)$ and further activates the following Akt protein [34,39-45]. PI3K $\alpha$ and mitogen-activated protein kinase (MAPK) signaling are both mediated by K-Ras4B. However, Saur et al. have recently revealed that in K-Ras4B-driven adenocarcinoma, PI3K $\alpha$ signaling is abnormally stimulated by the mutated K-Ras4B while the MAPK pathway cannot be activated [21]. Hence, both $\mathrm{CaM}$ and PI3K $\alpha$ are regarded as important regulators in the K-Ras4B-driven adenocarcinoma. Additionally, previous research has shown that CaM can directly interact with PI3K $\alpha$ through its $p 85 \alpha$ subunit and enhance its activity [46]. Thus, based on these results, we have recently put forward a ternary complex model in which the coordination of CaM and PI3K $\alpha$ with K-Ras4B forms a CaM-PI3K $\alpha-K-$ Ras4B ternary complex, which leads to PDAC [47-49]. According to this hypothesis, CaM-PI3K $\alpha-\mathrm{K}-$ Ras4B interact with each other, which explains the above observation that both $\mathrm{CaM}$ and PI3K $\alpha$ modulate the K-Ras4B-driven carcinogenesis. However, the detailed interactions between $\mathrm{CaM}$ and $\mathrm{PI} 3 \mathrm{~K} \alpha$ are still unknown.

Here, we explored the two regulatory components of the ternary complex, CaM and PI3K $\alpha$. We employed the molecular modeling and molecular dynamics (MD) simulations to explore the interactions between $\mathrm{CaM}$ and both the $\mathrm{cSH} 2$ and $\mathrm{nSH} 2$ domains of $\mathrm{p} 85 \alpha$ regulatory subunit of $\mathrm{PI} 3 \mathrm{~K} \alpha$. We first demonstrated that CaM can replace pEGFR to bind to the cSH2 and nSH2 domains. The potential models for the CaM-cSH2 and CaM-nSH2 interactions were unmasked. Detailed interactions of the CaM-cSH2 and CaM-nSH2 complexes were further revealed, which was applied to construct a complete CaM-PI3K $\alpha$ complex. The obtained results not only offer a support for our previous ternary complex hypothesis but also provide a structural basis for future drug design targeting CaM-PI3K $\alpha$ protein-protein interactions.

\section{Results}

\subsection{Overview of the Complex Structures}

200 ns MD simulations were performed for the ten complex systems, including C1-C4 for CaM-cSH2, N1-N4 for CaM-nSH2, pEGFR-cSH2, and pEGFR-nSH2. We first had a brief overview of the conformation of the complexes after simulations and found that CaM can bind to the $\mathrm{c} / \mathrm{nSH} 2$ domains in different topologies. For the cSH2 systems, $\mathrm{CaM}$ is in a collapsed conformation in $\mathrm{C} 1$, while in C2-C4 it adopts a stretched conformation (Figure 1). For the nSH2 systems, CaM presents a collapsed conformation in N1, N3 and N4, while in N2 it takes on a stretched conformation which enables it to wrap around nSH2 tightly (Figure 2).

The $\mathrm{C} \alpha$ atoms root-mean-square deviation (RMSD) of the trajectories with respect to the original structures was calculated to reveal the dynamic conformational changes throughout the MD process. A relatively unchanged RMSD reflects the equilibrium of the simulation system and the following analysis of the trajectories only focuses on the trajectories that reach equilibrium. The equilibrium time for C1-C4 and pEGFR-cSH2 is 60, 114, 10, 60 and 30 ns, respectively, (Figure 3A) and for N1-N4 and pEGFR-nSH2, it is 20, 40, 10, 10 and 30 ns, respectively (Figure 3B). 


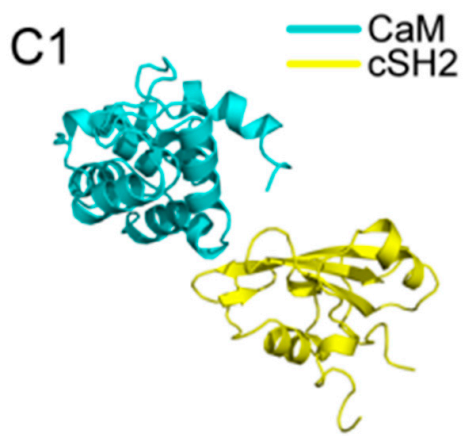

$\mathrm{C} 2$

C3

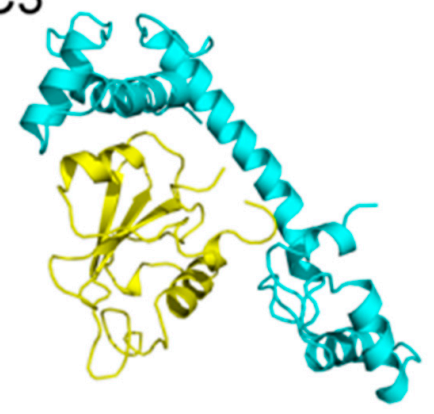

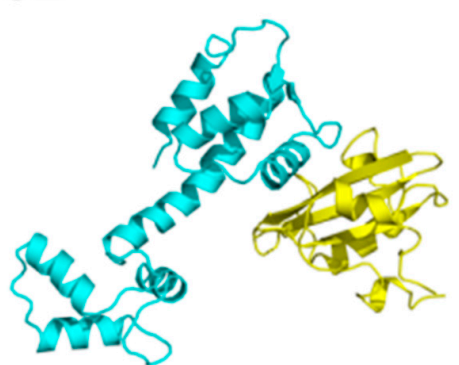

C4

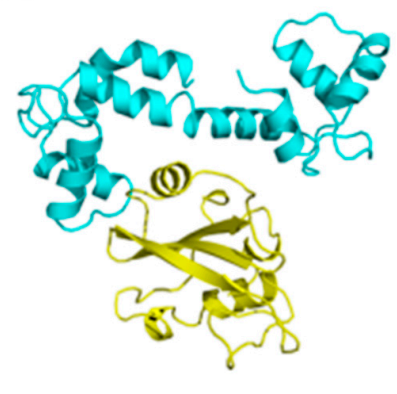

Figure 1. Structures of (C1-C4) complexes after 200 ns MD (molecular dynamics) simulations. Cyan structure represents $\mathrm{CaM}$, and yellow for cSH2.
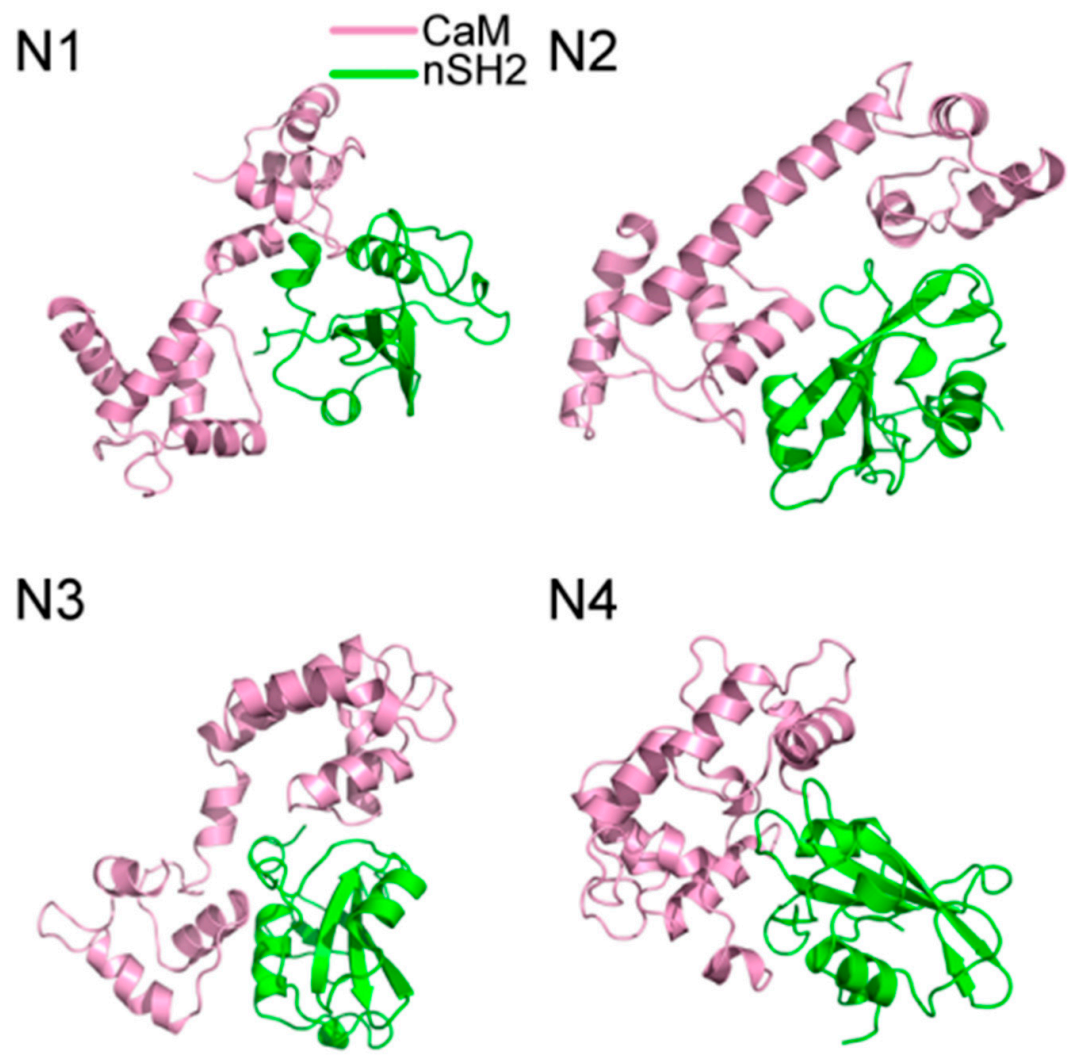

Figure 2. Structures of (N1-N4) complexes after 200 ns MD simulations. Pink structure represents CaM, and green for $\mathrm{nSH} 2$. 
(A)
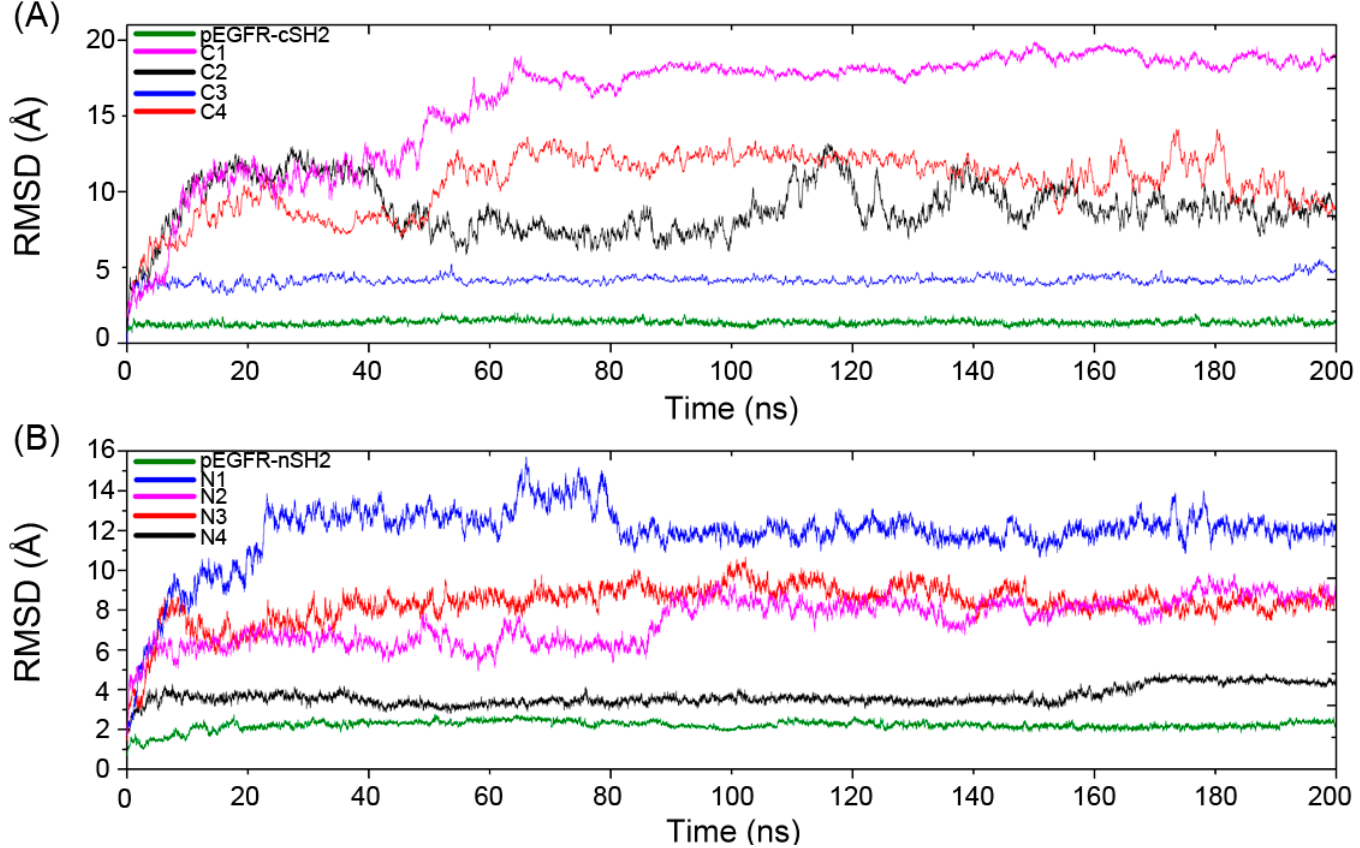

Figure 3. The $\mathrm{C} \alpha$ atoms root-mean-square deviation (RMSD) of eight CaM-p85 $\alpha$ and two pEGFR-p85 $\alpha$ systems along 200 ns MD simulations. (A) RMSD for CaM-cSH2 and pEGFR-cSH2 systems. (B) RMSD for CaM-nSH2 and pEGFR-nSH2 systems.

The RMSDs in C1 and N1 are relatively large and the influence of CaM topology on RMSD contradicted with previous study. Jang et al. reported that the stretched CaM structure would yield a large RMSD while the collapsed one was related to a small value [50]. However, in our simulations, such relationship is not obvious. In C1, the collapsed CaM produces a large RMSD, while in N2 the stretched CaM produces a relatively small RMSD. Such phenomena can be explained by different interaction modes of $\mathrm{CaM}-\mathrm{cSH} 2$ and $\mathrm{CaM}-\mathrm{nSH} 2$. During the simulation process, $\mathrm{CaM}$ underwent movement or rotation around its binding site on $\mathrm{c} / \mathrm{nSH} 2$, which would lead to a large RMSD for the binary complexes of CaM-cSH2 and CaM-nSH2.

\subsection{MM/GBSA Free Energy Analysis}

To understand the energetics of the interaction between CaM or pEGFR and c/nSH2 domains, binding free energy $\left(\Delta \mathrm{G}_{\text {binding }}\right.$ ) of $\mathrm{CaM}$ or pEGFR to $\mathrm{cSH} 2$ or $\mathrm{nSH} 2$ was calculated using MM/GBSA method. As shown in Table 1, C2, C3, N2, and N3 all underwent comparable free energy changes to the pEGFR systems during binding process and the binding free energy of $\mathrm{N} 4$ was even lower than the pEGFR system. Insights into the compositions of the interaction energy revealed that the major contribution originated from the electrostatic term. The similar or even lower binding free energy suggests that $\mathrm{CaM}$ can replace pEGFR and interact with $\mathrm{c} / \mathrm{nSH} 2$ domains, which supports the ternary complex hypothesis.

To quantify the contributions of different residues to the binding free energy in details, the total energy change was decomposed into each residue by weight. Residues with free energy contributions lower than $-0.3 \mathrm{kcal} / \mathrm{mol}$ were considered to be critical to the protein-protein interaction. Most of these important residues are located at the interfaces of CaM-cSH2 and CaM-nSH2 or pEGFR-cSH2 and pEGFR-nSH2, implying their pivotal roles in the protein-protein interaction. These important residues from the $\mathrm{c} / \mathrm{nSH} 2$ domains reflect the effect exerted by CaM or $\mathrm{pEGFR}$. We further aligned the amino acids sequences to compare the different important residues in different systems (Table 2, Figure S1). If the important residues in the CaM systems largely overlap with the ones in the pEGFR systems, it indicates that the corresponding CaM may have a pEGFR-like mode. As is shown in Table 2, 
$\mathrm{C} 2$ and N2 have the most overlapping residues with the corresponding pEGFR-p85 $\alpha$ complex systems. In C2 there are 8 overlapping residues (Figure S1A), while in N2 there are 11 (Figure S1B). Most of these important residues are located along the interface between CaM or pEGFR and c/nSH2, suggesting that they are involved in the protein-protein interactions. More overlapping residues in the C2 and $\mathrm{N} 2$ also implies that the CaM in these two systems imposes a similar influence as pEGFR. Hence, it is likely that CaM can replace and mimic pEGFR's role to interact with the $\mathrm{c} / \mathrm{nSH} 2$ domains in the C2 and N2 styles, respectively.

Table 1. Free energy analysis $(\mathrm{kcal} / \mathrm{mol})$ for the CaM/pEGFR-p85 $\alpha$ interactions ${ }^{\mathrm{a}}$.

\begin{tabular}{|c|c|c|c|c|c|}
\hline Systems & $\mathrm{C} 1$ & $\mathrm{C} 2$ & $\mathrm{C} 3$ & $\mathrm{C} 4$ & pEGFR-cSH2 \\
\hline $\mathrm{DE}_{\text {ele }}$ & $-487.24(72.84)$ & $-454.04(56.60)$ & $-481.67(72.71)$ & $-648.54(67.12)$ & $-272.97(176.16)$ \\
\hline $\mathrm{DE}_{\mathrm{vdW}}$ & $-28.32(8.80)$ & $-52.71(5.67)$ & $-104.44(9.22)$ & $-44.78(8.30)$ & $-26.16(18.75)$ \\
\hline $\mathrm{DG}_{\text {nonpolar }}$ & $-4.84(1.46)$ & $-7.08(0.98)$ & $-14.41(1.35)$ & $-6.74(1.05)$ & $-4.72(3.31)$ \\
\hline $\mathrm{DG}_{\text {polar }}$ & $498.13(73.12)$ & $476.20(53.19)$ & $546.28(67.34)$ & $677.47(67.24)$ & $264.58(170.14)$ \\
\hline $\mathrm{DG}_{\text {binding }}$ & $-17.43(11.54)$ & $-30.56(10.19)$ & $-39.83(10.46)$ & $-15.84(7.87)$ & $-34.56(24.51)$ \\
\hline Systems & N1 & N2 & N3 & N4 & pEGFR-nSH2 \\
\hline $\mathrm{DE}_{\text {ele }}$ & $-327.90(66.64)$ & $-696.61(78.38)$ & $-445.38(102.15)$ & $-750.52(89.50)$ & $-420.68(242.11)$ \\
\hline $\mathrm{DE}_{\mathrm{vdW}}$ & $-73.53(9.76)$ & $-78.07(13.17)$ & $-101.85(11.04)$ & $-132.88(10.91)$ & $-40.35(23.74)$ \\
\hline $\mathrm{DG}_{\text {nonpolar }}$ & $-11.06(1.25)$ & $-12.18(1.79)$ & $-14.69(1.73)$ & $-20.70(1.41)$ & $-6.71(3.88)$ \\
\hline $\mathrm{DG}_{\text {polar }}$ & $370.08(62.20)$ & 718.05 (75.83) & $484.00(98.17)$ & $787.72(86.24)$ & $395.50(226.89)$ \\
\hline $\mathrm{DG}_{\text {binding }}$ & $-31.35(10.49)$ & $-56.62(10.88)$ & $-63.23(12.84)$ & $-95.68(11.78)$ & $-65.53(38.63)$ \\
\hline
\end{tabular}

Table 2. Comparison of the important residues for CaM/pEGFR-p85 $\alpha$ interactions.

\begin{tabular}{ccccc}
\hline System & C1 & C2 & C3 & C4 \\
\hline Overlap residues & 5 & 8 & 4 & 3 \\
\hline System & N1 & N2 & N3 & N4 \\
\hline Overlap residues & 5 & 11 & 5 & 5 \\
\hline
\end{tabular}

* Systems with asterisks are the ones with most overlapping residues important for interactions.

\subsection{Superposition Analysis of the Candidate Structures}

MM/GBSA analysis revealed that replacement of pEGFR by CaM is energetically favorable and the decomposition results showed that CaM in the $\mathrm{C} 2$ and $\mathrm{N} 2$ may function in a similar manner to pEGFR. To further validate these results, superposition analysis of ten complexes after simulations was carried out. C1-C4 were superposed to the pEGFR-cSH2 complex (Figure 4A,C) and N1-N4 to the pEGFR-nSH2 complex (Figure 4B,D). The results showed that CaM can bind to $\mathrm{CSH} 2$ or $\mathrm{nSH} 2$ at different sites. However, only in C2 and N2 systems the binding sites of CaM overlap with those of pEGFR (Figure 4A,B), which is in agreement with the previous energy decomposition results.

In C2, CaM takes on a stretched conformation, with part of its N-lobe, helix and random structure (P44-G62) inserting into the pEGFR site (Figure 5). These structures interact with the cSH2 in a similar topology to pEGFR, which can account for their similar effect. CaM binds to cSH2 near the pEGFR site and therefore can mimic the effect induced by pEGFR. In N2, CaM is in an extended conformation and it closely wraps around $\mathrm{nSH} 2$ and overlaps with the pEGFR binding site. The stretched linker of $\mathrm{CaM}$ in this system hangs across the pEGFR binding pocket and the helices in C-lobe (Y139-A148) and N-lobe (T45-D59) also interact with the pEGFR site (Figure 6). 
A



C

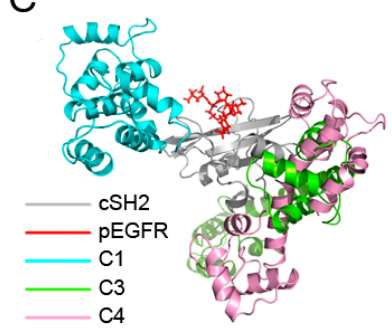

B

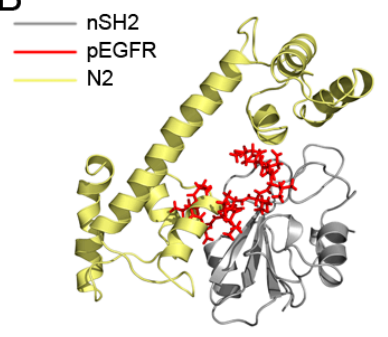

D

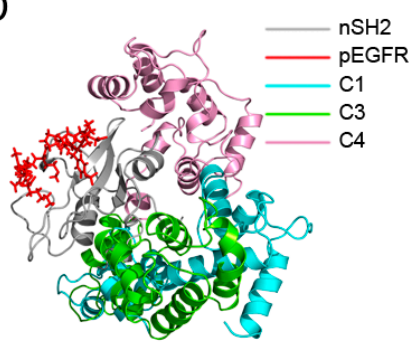

Figure 4. Overview of the superposition structures after $200 \mathrm{~ns}$ simulations. (A) Superposition of the structures of $\mathrm{C} 2$ and pEGFR-cSH2; (B) Superposition of the structures of N2 and pEGFR-nSH2; (C) Superposition of the structures of $\mathrm{C} 1, \mathrm{C} 3, \mathrm{C} 4$ and pEGFR-cSH2; (D) Superposition of the structures of N1, N3, N4 and pEGFR-nSH2. c/nSH2, pEGFR, C1/N1, C2/N2, C3/N3, and C4/N4 are colored in gray, red, cyan, pale yellow, chartreuse, and pink, respectively.

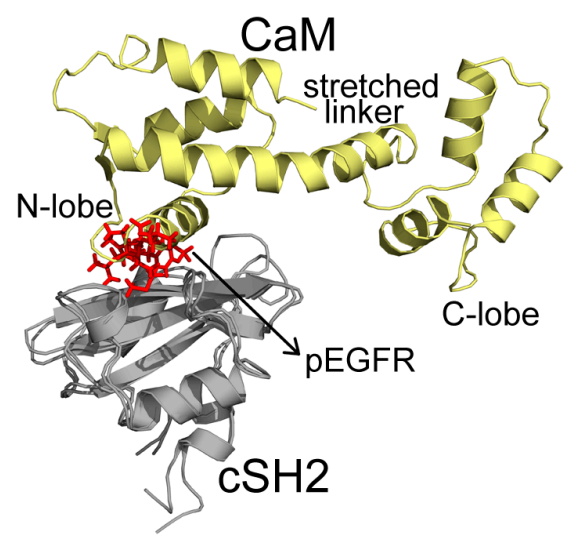

Figure 5. Superposition structures of $\mathrm{C} 2$ and pEGFR-cSH2 after $200 \mathrm{~ns}$ simulations. $\mathrm{CaM}, \mathrm{cSH} 2$, and pEGFR are colored in pale yellow, gray, and red, respectively. Important parts of CaM structures are specified.

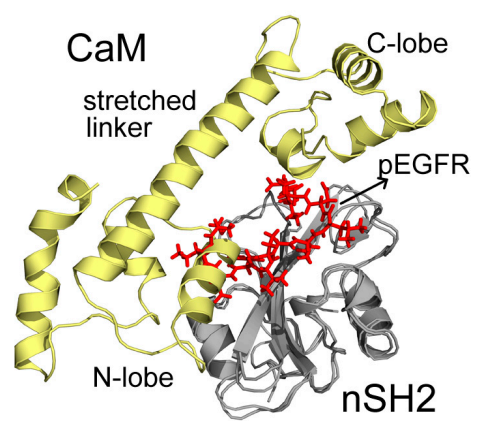

Figure 6. Superposition structures of $\mathrm{N} 2$ and pEGFR-nSH2 after 200 ns simulations. CaM, nSH2, and pEGFR are colored in pale yellow, gray, and red, respectively. Important parts of CaM structures are specified. 


\subsection{Insights into the Detailed $\mathrm{CaM}-\mathrm{cSH} 2$ and $\mathrm{CaM}-n \mathrm{SH} 2$ Interactions}

Structural analysis showed that in C2 and N2, the binding modes of CaM are similar to the ones of pEGFR. PISA (Proteins, Interfaces, Structures and Assemblies) [51] was then employed to explore the detailed interactions of the two systems through analysis of their interfaces.

In $\mathrm{C} 2$, the binding interface of $\mathrm{CaM}$ and $\mathrm{CSH} 2$ is $539.1 \AA^{2}$, deriving from 13 residues of $\mathrm{CaM}$ and 21 residues of cSH2. For N2, the interface is $1217.6 \AA^{2}$, consisting of 34 residues of CaM and 35 residues of $\mathrm{nSH} 2$. Similar to the free energy decomposition analysis, the interface residues on the $\mathrm{c} / \mathrm{nSH} 2$ domains from the C1-C4 and N1-N4 were compared with those in pEGFR systems (Table 3, Figure S2). The interface residues in C2 and N2 significantly overlap with those in pEGFR-cSH2 and pEGFR-nSH2, which is consistent with the energy decomposition results. In addition, the interface residues detected by PISA in C2 and N2 also overlaps with those obtained from the free energy decomposition analysis.

Table 3. Comparison of the interface residues of CaM/pEGFR-p85 $\alpha$ interactions.

\begin{tabular}{ccccc}
\hline System & C1 & C2 & C3 & C4 \\
\hline Overlap residues & 2 & 14 & 0 & 0 \\
\hline System & N1 & N2 & N3 & N4 \\
\hline Overlap residues & 0 & 15 & 2 & 5 \\
\hline tems with asterisks are the ones with most overlapping interface residues.
\end{tabular}

In addition to interface areas and residues, PISA also uncovered the molecular interactions along the binding interfaces. In $\mathrm{C} 2$, there are 5 inter-molecular hydrogen bonds and 4 salt bridges across the interface (Table S1). D47 of CaM makes salt bridges with the protonated nitrogen in the guanidine group of $\mathrm{R} 37$ of cSH2 and E79 of CaM forms another salt bridge with the amino group of $\mathrm{K} 41$ of cSH2. G56 of CaM is hydrogen-bonded to R19 of cSH2 (Figure 7). In N2, there exist 15 hydrogen bonds and 10 salt bridges between CaM and nSH2 (Figure 8, Table S2). Hotspot residues on CaM such as D54 and E41 form salt bridges with 3 neighboring residues from $\mathrm{nSH} 2$, revealing their critical roles within the complex. The pivotal residues involved in multiple inter-protein interactions play central parts in the binding of CaM to p $85 \alpha$ subunit and may become potential targets for modulation of the CaM-PI3K $\alpha$ protein-protein interactions.

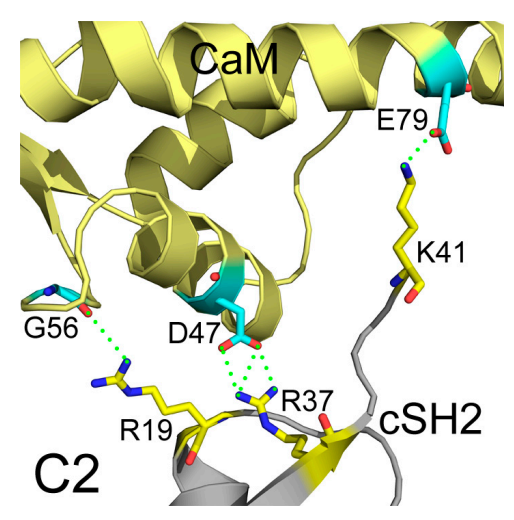

Figure 7. Detailed interactions between $\mathrm{CaM}$ and cSH2 in $\mathrm{C} 2$ after $200 \mathrm{~ns}$ simulation. Hydrogen bonds are shown as green dashed lines. CaM structure is colored in pale yellow, and cSH2 structure is in gray. $\mathrm{CaM}$ residues involved in hydrogen bonds formation are in cyan, and the cSH2 ones are in yellow. 




Figure 8. Detailed interaction between CaM and nSH2 in N2 after 200 ns simulation. Hydrogen bonds are depicted by green dashed lines. CaM structure is colored in pale yellow, and $\mathrm{nSH} 2$ structure is in gray. $\mathrm{CaM}$ residues involved in hydrogen bonds formation are in cyan, and the $\mathrm{nSH} 2$ ones are in yellow.

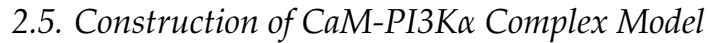

The binding modes of $\mathrm{CaM}-\mathrm{cSH} 2$ and $\mathrm{CaM}-\mathrm{nSH} 2$ shed light on the detailed interactions between $\mathrm{CaM}$ and the $\mathrm{p} 85 \alpha$ regulatory subunit of PI3K $\alpha$. To have a view of the complete CaM-PI3K $\alpha$ interaction, we constructed the full-length CaM-PI3K $\alpha$ complex model. According to previous analysis, $\mathrm{C} 2$ and N2 are regarded as the ideal modes for $\mathrm{CaM}$ to interact with $\mathrm{c} / \mathrm{nSH} 2$ in the $\mathrm{p} 85 \alpha$ subunit of PI3K $\alpha$, so their protein complexes before $200 \mathrm{~ns}$ simulations were used to assemble our model. Crystal structure of PI3K $\alpha$ (PDB ID: 4OVV) [52] was extracted from the PDB and the C2 and N2 complexes were aligned to it via structural superimposition on the $\mathrm{c} / \mathrm{nSH} 2$ domains. After constructing the primary CaM-PI3K $\alpha$ complex model, energy optimization of the overall systems was performed.

As shown in Figure 9, CaM takes on an extended conformation to interact with $\mathrm{p} 85 \alpha$ in PI3K $\alpha$ at the cSH2 domain. CaM places its N-lobe on the $\mathrm{CSH} 2$ surface and exerts a pEGFR-like effect. However, both the stretched linker and C-lobe of CaM project away, without any contact with other parts of PI3K $\alpha$. Also, in our model there exists limited space on $\mathrm{CSH} 2$ for CaM binding, which results in the narrow interface between $\mathrm{CaM}$ and $\mathrm{cSH} 2$. The relatively small interface may be one of the reasons for the movement of CaM throughout the interaction process and more importantly, it explains why an allosteric mechanism may be involved in the activation of PI3K $\alpha$ by CaM through cSH2 [18]. Given the narrow interface between $\mathrm{CaM}$ and $\mathrm{cSH} 2$, direct protein-protein contact may not be able to exert effects strong enough and hence, allosteric signaling may play a role in the activation of PI3K $\alpha$ by $\mathrm{CaM}$ through $\mathrm{cSH} 2$. As for the $\mathrm{nSH} 2$ domain, previous comparison of $\mathrm{N} 2$ structures before and after simulation shows that $\mathrm{CaM}$ moved along the $\mathrm{nSH} 2$ interface during simulation and this observation can find its origin in our assembly. In our model, the structures near $\mathrm{nSH} 2$ are relatively flexible and the inserting CaM does not have much conformational hindrance around its binding site. Hence, it is 
very likely that when interacting with full-length $\mathrm{PI} 3 \mathrm{~K} \alpha \mathrm{CaM}$ will also move across the nSH2 interface, inserting towards the inner part of $\mathrm{PI} 3 \mathrm{~K} \alpha$ and impose its activating effect. Moreover, $\mathrm{nSH} 2$ situates near iSH2 domain (part of p85 $\alpha$ subunit that connects $\mathrm{nSH} 2$ to $\mathrm{cSH} 2$ ) and $\mathrm{p} 110 \alpha$ subunit of PI3K $\alpha$ and the CaM binding site locates adjacent to the interface between p110 $\alpha$ and nSH2. Since there are relatively fewer conformational constraints around the interfaces, these observations support our simulation results, in which CaM gradually "crawls" across nSH2 and inserts into the whole protein assembly to exert its influence.
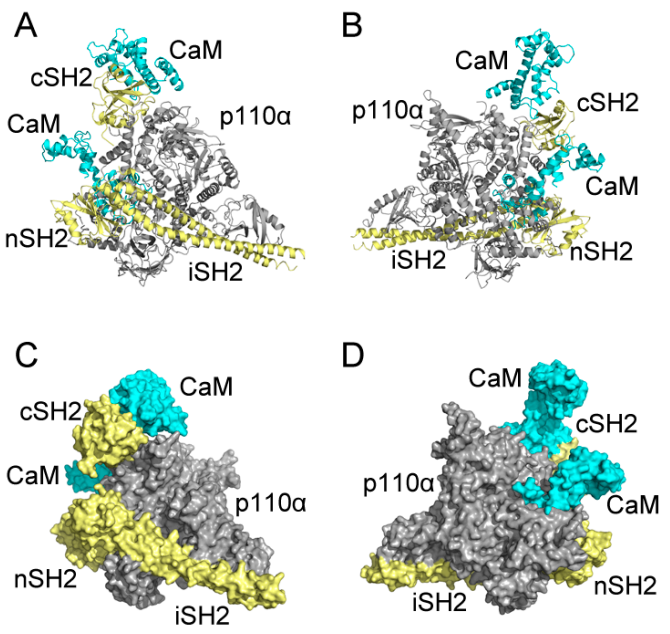

Figure 9. Predicted CaM-PI3K $\alpha$ complex model constructed with CaM in C2 and N2 and full-length Phosphatidylinositol 3-Kinase $\alpha(\mathrm{PI} 3 \mathrm{~K} \alpha)$. Complex system in cartoon mode from the front (A) and back (B); and complex system in surface mode from the front (C) and back (D). CaM is in cyan, and p110 $\alpha$ subunit of $\mathrm{PI} 3 \mathrm{~K} \alpha$ is in gray, and $\mathrm{p} 85 \alpha$ subunit, including $\mathrm{cSH} 2, \mathrm{nSH} 2$ and iSH2 are in pale yellow.

\section{Discussion}

CaM and PI3K $\alpha$ are known to regulate several cellular events [22-24,53]. In addition, there exists crosstalk between them and their related intracellular pathways. Under normal circumstances, PI3K $\alpha$ is activated by $\mathrm{pEGFR}$ and K-Ras4B together. pEGFR binds to $\mathrm{c} / \mathrm{nSH} 2$ domains of the $\mathrm{p} 85 \alpha$ regulatory subunit of $\mathrm{PI} 3 \mathrm{~K} \alpha$, which can relieve the inhibitory effect of $\mathrm{p} 85 \alpha$ on its $\mathrm{p} 110 \alpha$ catalytic subunit. After that, K-Ras4B binds to PI3K $\alpha$ and allosterically stimulates the production of PIP 3 [47,48]. Previously, CaM has been reported to be able to activate PI3K $\alpha$ through $\mathrm{p} 85 \alpha$ subunit independent of K-Ras4B [45,46], and recent research suggests that CaM can replace pEGFR and activate PI3K $\alpha$ aberrantly [47-49]. However, details behind this interaction still remain elusive. Unraveling the mechanisms underlying CaM-PI3K $\alpha$ crosstalk through which CaM abnormally replaces pEGFR and activates $\mathrm{PI} 3 \mathrm{~K} \alpha$ is expected to contribute to future research and clinical application.

Here, by MD simulations we found that CaM can bind to $\mathrm{c} / \mathrm{nSH} 2$ domains with comparable reaction free energy to $\mathrm{pEGFR}$, implying that the replacement of $\mathrm{pEGFR}$ by CaM is feasible. Through structure superposition and interface analysis, C2 complex and N2 complex were predicted to be the ideal models for CaM-cSH2 and CaM-nSH2 interactions, in which CaM adopts similar acting modes to pEGFR. The details of the interactions in these two systems including hydrogen bonds and salt bridges were unmasked, and they are the origin of the tight binding of CaM and its ability to induce the pEGFR-like effect. The hotspot residues for CaM-cSH2 and CaM-nSH2 interactions were identified, which will be instructive to future related study. Moreover, based on these findings we constructed a CaM-PI3K $\alpha$ complex assembly, which provides guidance for further exploration. Similarly, Zhang et al. also explored the crosstalk between CaM and PI3K lately, but they focus on phosphorylated CaM at Y99 [45]. Both of our studies produce similar results supporting each other, in which the CaM takes on similar topologies to interact with $\mathrm{PI} 3 \mathrm{~K} \alpha$ and exerts the activating effect. 
Our research supplies in-depth insights into the CaM-PI3K $\alpha$ crosstalk, and will be of great significance to future relevant research and application.

Recently, we put forward a novel theory about the CaM-PI3K $\alpha$ interaction where K-Ras4B is also involved and they form a ternary complex together, especially in carcinogenesis [47-49]. Details of this hypothesis remain unresolved and this gap can be partly filled by our research, which provides underlying mechanisms of the $\mathrm{CaM}-\mathrm{cSH} 2$ and $\mathrm{CaM}-\mathrm{nSH} 2$ interactions. Moreover, our findings not only supply solid support for this hypothesis, but also provide instructions for therapies towards the K-Ras4B-driven adenocarcinoma regulated by CaM and PI3K $\alpha$. Modulations of protein-protein interaction (PPI) have already become a promising idea in drug development [13,47,54,55], and PPIs inhibitors as well as related drugs can be designed based on the interaction modes and hotspot residues uncovered here. Very recently, we have found some potential allosteric targets on Ras [47,56-59]. Combining our previous findings and the discovery here, we will be able to target the ternary complex, which can prevent the oncogenesis of the K-Ras4B-driven adenocarcinoma. Hence, our study here provides crucial theoretical basis for further research on CaM and PI3K $\alpha$ as well as sheds light on future clinical adenocarcinoma therapy and drug development.

\section{Materials and Methods}

\subsection{Construction of Simulation Systems}

For the pEGFR-nSH2 system, the crystal structure of nSH2 domain of the $\mathrm{p} 85 \alpha$ regulatory subunit of PI3K $\alpha$ and platelet-derived growth factor receptor (PDGFR) peptide (PDB ID: 2IUI), which can mimic the domain of pEGFR that interacts with the nSH2 domain of PI3K $\alpha$ [60], was extracted from the RCSB Protein Data Bank (PDB). For the PDGFR peptide and PI3K $\alpha$ cSH2 system, $1 \mathrm{H} 9 \mathrm{O}$ was chosen from the PDB [61].

As for the CaM-cSH2 and CaM-nSH2 systems, no structures are available currently. PRISM [62-64] was employed to carry out the inter-protein interaction prediction to set up the complex systems for further MD simulations. PRISM compared patches along the surfaces of the two interacting proteins, and if they had similar structural features to two complementary sides of a template interface, and the "hot spot" residues on both the proteins and templates were evolutionarily conserved, these two proteins would be regarded to be likely to interact with each other. Such comparison not only considered the rigid structure similarity, but also took the backbone and side-chain flexibility into account $[63,64]$. Finally, such kind of interaction would be graded according to the binding free energy and the different contribution terms of it, including desolvation energy, van der Waals interactions, partial electrostatics and so on $[65,66]$. We first downloaded all the available structures of CaM, cSH2, and $\mathrm{nSH} 2$ from PDB, and PRISM prediction was then carried out based on these structural data. $\mathrm{CaM}$ was docked to $\mathrm{CSH} 2$ and $\mathrm{nSH} 2$ respectively, and the top four complexes with highest PRISM scores in these two systems were chosen, and would be further simulated and analyzed [67-69]. These eight complexes structures were denoted as the $\mathrm{C} 1$ to $\mathrm{C} 4$ and $\mathrm{N} 1$ to $\mathrm{N} 4$ for the $\mathrm{CSH} 2$ and nSH2 systems, respectively. The compositions of these systems are shown in Table 4 and MD simulations were then performed.

Table 4. Compositions of the predicted CaM-cSH2 and CaM-nSH2 complexes by PRISM.

\begin{tabular}{ccccc}
\hline System & C1 & C2 & C3 & C4 \\
\hline CaM & 1CDL * & 1CLL & 1CLL & 1CLL \\
cSH2 & 1H9O & 1H9O & 1H9O & 1H9O \\
\hline System & N1 & N2 & N3 & N4 \\
\hline CaM & 2BE6 & 1S26 & 2BE6 & 2BE6 \\
nSH2 & 2IUG & 2IUH & 2IUG & 2IUH \\
\hline \multicolumn{5}{c}{ The PDB ID of the protein structure. }
\end{tabular}




\subsection{Simulations}

MD simulations for the ten systems described above (C1-C4, N1-N4, pEGFR-cSH2, and pEGFR-nSH2) were carried out using Amber 14 (San Francisco, CA, USA) [70]. The modified ff03 force field [71] was applied to the calculation of the force field parameters of the complex systems. All the complexes above first underwent solvation using the TIP3P water model, and $\mathrm{Na}^{+}$counterions were added to neutralize the whole systems. The energy of the systems was minimized after the preparation process. The energy minimization could be divided into two parts, and in the first part, the proteins structures were held in place, and 5000 steps of minimization cycles were carried out to minimize the energy of the water molecules and the counter ions. After that, complexes were relaxed and the second round of minimization without restriction followed. Once the system energy had been minimized, they were heated from 0 to $300 \mathrm{~K}$ within $300 \mathrm{ps}$, under $10 \mathrm{kcal} /\left(\mathrm{mol} \times \AA^{2}\right)$ positional restraint in a canonical ensemble (NVT), and then equilibration of the system was carried out at the target temperature, $300 \mathrm{~K}$, under the same conditions for $700 \mathrm{ps}$. Finally, all the complexes underwent $200 \mathrm{~ns}$ MD simulations in isothermal and isobaric ensemble with periodic boundary conditions. Long-range electrostatic interactions were solved with the help of the particle mesh Ewald method, and a $10 \AA$ cut-off was employed to deal with the short-range electrostatics and van der Waals interactions. Within systems, all hydrogen-involved covalent bonds were restricted by the SHAKE method, and the final trajectories were written out every 20 ps.

\subsection{Molecular Mechanics Generalized Born Surface Area Calculations}

In Amber 14 [70], plugin MMPBSA.py was used to carry out the Molecular Mechanics Generalized Born Surface Area (MM/GBSA) calculations. Free energy was calculated for the whole complex, receptor $(\mathrm{CaM})$, and ligand (nSH2, $\mathrm{CSH} 2$ or pEGFR) correspondingly. The free energy of the binding reaction came as a result of the following Equation (1):

$$
\Delta \mathrm{G}=\mathrm{G}_{\text {complex }}-\mathrm{G}_{\text {receptor }}-\mathrm{G}_{\text {ligand }}
$$

In Equation (1), free energy (G) was calculated according to Equation (2):

$$
\Delta \mathrm{G}=\Delta \mathrm{E}_{\text {gas }}+\Delta \mathrm{G}_{\text {solvation }}-\mathrm{T} \Delta \mathrm{S}
$$

In which, $\Delta \mathrm{E}_{\text {gas }}$ represented the gas phase molecular mechanical energy, $\Delta \mathrm{G}_{\text {solvation }}$ stood for the solvation free energy, and $-\mathrm{T} \Delta \mathrm{S}$ for the entropy term. $\Delta \mathrm{E}_{\text {gas }}$ could be further divided into three parts, as is shown in Equation (3), and it equaled to the sum of the van der Waals energy $\left(\Delta \mathrm{E}_{\mathrm{vdW}}\right)$, electrostatic energy $\left(\Delta \mathrm{E}_{\text {ele }}\right)$ and gas phase internal energy $\left(\Delta \mathrm{E}_{\text {int }}\right)$ :

$$
\Delta \mathrm{E}_{\text {gas }}=\Delta \mathrm{E}_{\mathrm{vdW}}+\Delta \mathrm{E}_{\text {ele }}+\Delta \mathrm{E}_{\text {int }}
$$

Continuum solvent methods were employed to calculate $\Delta G_{\text {solvation, which consisted of polar }}$ contribution $\left(\Delta \mathrm{G}_{\mathrm{PB} /}\right)$ and non-polar contribution $\left(\Delta \mathrm{G}_{\text {nonpolar }}\right)$, given as Equation (4):

$$
\Delta \mathrm{G}_{\text {solvation }}=\Delta \mathrm{G}_{\mathrm{PB}}+\Delta \mathrm{G}_{\text {nonpolar }}
$$

Calculation of the electrostatic solvation energy was based on the finite difference PB model, in which the solute and water dielectric constants were chosen to be 1 and 80 respectively. Equation (5) was used to work out the non-polar contribution $\left(\Delta G_{\text {nonpolar }}\right)$ to the solvation free energy $\left(\Delta G_{\text {solvation }}\right)$ :

$$
\Delta \mathrm{G}_{\text {solvation }}=\gamma \mathrm{SASA}+\mathrm{b}
$$

In Equation (5), solvent-accessible surface-area was abbreviated as $S A S A$, solvation parameter $\gamma$ equaled $0.00542 \mathrm{kcal}\left(\mathrm{mol}^{-1} \cdot \AA^{-2}\right)$ and the other solvation parameter, b, was $0.92 \mathrm{kcal} / \mathrm{mol}$. 
The conformation entropy $(-\mathrm{T} \Delta \mathrm{S})$ was omitted in our calculations. This term is usually estimated with quasi harmonic analysis of the simulation trajectories using normal mode analysis, which demands a lot of computational time. Since in our study, we only focused on the relative ordering of binding free energy, and throughout the interaction process, the interaction modes and the overall root-mean-square deviation (RMSD) of the $\mathrm{C} \alpha$ atoms with respect to the original structures during simulations did not change significantly. Taking all these into consideration, we left out the conformation entropy term in our free energy calculation. The overall free energy difference $\Delta G$ was distributed to every residue within the system by weight with the help of the MM/GBSA method in Amber 14.

\section{Conclusions}

CaM-PI3K $\alpha$ crosstalk is a common and central process within series of cellular activities and human physiological or pathological conditions. Here in our study, using MD simulations and molecular modeling, we explored into the detailed interaction modes between CaM and the cSH2 and $\mathrm{nSH} 2$ domains of the $\mathrm{p} 85 \alpha$ regulatory subunit in PI3K $\alpha$. We first demonstrated that CaM can interact with these two domains from an energetic view and by structural superposition we found out the potential ideal binding poses for CaM to interact with $\mathrm{cSH} 2$ and $\mathrm{nSH} 2$. Detailed inter-molecular interactions such as salt bridges and hydrogen bonds were revealed and the corresponding hotspot residues for protein-protein interactions were also unmasked. With these finding, we also assembled the full-length CaM-PI3K $\alpha$ complex, which is the first report for this interaction system. Our study not only provides solid theoretical basis for relevant research but also shed light on related targeting drug discovery.

Supplementary Materials: Supplementary materials can be found at www.mdpi.com/1422-0067/19/1/151/s1.

Acknowledgments: This work was supported by National Basic Research Program of China (973 Program) (2015CB910403); National Natural Science Foundation of China (81603023, 81322046, 81302698, 81473137, 21778037); Shanghai Health and Family Planning Commission (20154Y0058).

Author Contributions: Shaoyong Lu and Jian Zhang conceived and designed the experiments; Duan Ni and Dingyu Liu performed the experiments; Duan Ni and Dingyu Liu analyzed the data; Shaoyong Lu and Jian Zhang contributed reagents/materials/analysis tools: All authors wrote the paper.

Conflicts of Interest: The authors declare no conflict of interest.

\section{References}

1. Barbacid, M. Ras genes. Annu. Rev. Biochem. 1987, 56, 779-827. [CrossRef] [PubMed]

2. Malumbres, M.; Barbacid, M. RAS oncogenes: the first 30 years. Nat. Rev. Cancer 2003, 3, 459-465. [CrossRef] [PubMed]

3. Karnoub, A.E.; Weinberg, R.A. Ras oncogenes: Split personalities. Nat. Rev. Mol. Cell Biol. 2008, 9, 517-531. [CrossRef] [PubMed]

4. Cox, A.D.; Der, C.J. Ras history: The saga continues. Small GTPases 2010, 1, 2-27. [CrossRef] [PubMed]

5. Bryant, K.L.; Mancias, J.D.; Kimmelman, A.C.; Der, C.J. KRAS: Feeding pancreatic cancer proliferation. Trends Biochem. Sci. 2014, 39, 91-100. [CrossRef] [PubMed]

6. Doma, E.; Rupp, C.; Baccarini, M. EGFR-ras-raf signaling in epidermal stem cells: Roles in hair follicle development, regeneration, tissue remodeling and epidermal cancers. Int. J. Mol. Sci. 2013, 14, 19361-19384. [CrossRef] [PubMed]

7. Downward, J. Targeting RAS signalling pathways in cancer therapy. Nat. Rev. Cancer 2003, 3, 11-22. [CrossRef] [PubMed]

8. Ledford, H. Cancer: The Ras renaissance. Nature 2015, 520, 278-280. [CrossRef] [PubMed]

9. Prior, I.A.; Lewis, P.D.; Mattos, C. A comprehensive survey of Ras mutations in cancer. Cancer Res. 2012, 72, 2457-2467. [CrossRef] [PubMed] 
10. Tomasini, P.; Serdjebi, C.; Khobta, N.; Metellus, P.; Ouafik, L.; Nanni, I.; Greillier, L.; Loundou, A.; Fina, F.; Mascaux, C.; et al. EGFR and KRAS Mutations Predict the Incidence and Outcome of Brain Metastases in Non-Small Cell Lung Cancer. Int. J. Mol. Sci. 2016, 17, 2132. [CrossRef] [PubMed]

11. Shen, Q.; Cheng, F.; Song, H.; Lu, W.; Zhao, J.; An, X.; Liu, M.; Chen, G.; Zhao, Z.; Zhang, J. Proteome-Scale Investigation of Protein Allosteric Regulation Perturbed by Somatic Mutations in 7,000 Cancer Genomes. Am. J. Hum. Genet. 2017, 100, 5-20. [CrossRef] [PubMed]

12. Pylayeva-Gupta, Y.; Grabocka, E.; Bar-Sagi, D. RAS oncogenes: Weaving a tumorigenic web. Nat. Rev. Cancer 2011, 11, 761-774. [CrossRef] [PubMed]

13. Gysin, S.; Salt, M.; Young, A.; McCormick, F. Therapeutic strategies for targeting ras proteins. Genes Cancer 2011, 2, 359-372. [CrossRef] [PubMed]

14. Stephen, A.G.; Esposito, D.; Bagni, R.K.; McCormick, F. Dragging ras back in the ring. Cancer Cell 2014, 25, 272-281. [CrossRef] [PubMed]

15. Carpelan-Holmstrom, M.; Nordling, S.; Pukkala, E.; Sankila, R.; Luttges, J.; Kloppel, G.; Haglund, C. Does anyone survive pancreatic ductal adenocarcinoma? A nationwide study re-evaluating the data of the Finnish Cancer Registry. Gut 2005, 54, 385-387. [CrossRef] [PubMed]

16. Lu, S.; Jang, H.; Nussinov, R.; Zhang, J. The Structural Basis of Oncogenic Mutations G12, G13 and Q61 in Small GTPase K-Ras4B. Sci. Rep. 2016, 6, 21949. [CrossRef] [PubMed]

17. Lu, S.; Banerjee, A.; Jang, H.; Zhang, J.; Gaponenko, V.; Nussinov, R. GTP Binding and Oncogenic Mutations May Attenuate Hypervariable Region (HVR)-Catalytic Domain Interactions in Small GTPase K-Ras4B, Exposing the Effector Binding Site. J. Biol. Chem. 2015, 290, 28887-28900. [CrossRef] [PubMed]

18. Nussinov, R.; Wang, G.; Tsai, C.J.; Jang, H.; Lu, S.; Banerjee, A.; Zhang, J.; Gaponenko, V. Calmodulin and PI3K Signaling in KRAS Cancers. Trends Cancer 2017, 3, 214-224. [CrossRef] [PubMed]

19. Wang, M.T.; Holderfield, M.; Galeas, J.; Delrosario, R.; To, M.D.; Balmain, A.; McCormick, F. K-Ras Promotes Tumorigenicity through Suppression of Non-canonical Wnt Signaling. Cell 2015, 163, 1237-1251. [CrossRef] [PubMed]

20. Nussinov, R.; Muratcioglu, S.; Tsai, C.J.; Jang, H.; Gursoy, A.; Keskin, O. The Key Role of Calmodulin in KRAS-Driven Adenocarcinomas. Mol. Cancer Res. 2015, 13, 1265-1273. [CrossRef] [PubMed]

21. Eser, S.; Reiff, N.; Messer, M.; Seidler, B.; Gottschalk, K.; Dobler, M.; Hieber, M.; Arbeiter, A.; Klein, S.; Kong, B.; et al. Selective requirement of PI3K/PDK1 signaling for Kras oncogene-driven pancreatic cell plasticity and cancer. Cancer Cell 2013, 23, 406-420. [CrossRef] [PubMed]

22. Stevens, F.C. Calmodulin: An introduction. Can. J. Biochem. Cell Biol. 1983, 61, 906-910. [CrossRef] [PubMed]

23. Chin, D.; Means, A.R. Calmodulin: A prototypical calcium sensor. Trends Cell Biol. 2000, 10, 322-328. [CrossRef]

24. Berchtold, M.W.; Villalobo, A. The many faces of calmodulin in cell proliferation, programmed cell death, autophagy, and cancer. Biochim. Biophys. Acta 2014, 1843, 398-435. [CrossRef] [PubMed]

25. Babu, Y.S.; Bugg, C.E.; Cook, W.J. Structure of calmodulin refined at 2.2 A resolution. J. Mol. Biol. 1988, 204, 191-204. [CrossRef]

26. Tidow, H.; Nissen, P. Structural diversity of calmodulin binding to its target sites. FEBS J. 2013, 280, 5551-5565. [CrossRef] [PubMed]

27. Vetter, S.W.; Leclerc, E. Novel aspects of calmodulin target recognition and activation. Eur. J. Biochem. 2003, 270, 404-414. [CrossRef] [PubMed]

28. Villalobo, A.; Garcia-Palmero, I.; Stateva, S.R.; Jellali, K. Targeting the calmodulin-regulated ErbB/Grb7 signaling axis in cancer therapy. J. Pharm. Pharm. Sci. 2013, 16, 177-189. [CrossRef] [PubMed]

29. Takemoto-Kimura, S.; Suzuki, K.; Horigane, S.I.; Kamijo, S.; Inoue, M.; Sakamoto, M.; Fujii, H.; Bito, H. Calmodulin kinases: Essential regulators in health and disease. J. Neurochem. 2017, 141, 808-818. [CrossRef] [PubMed]

30. Cui, C.; Merritt, R.; Fu, L.; Pan, Z. Targeting calcium signaling in cancer therapy. Acta Pharm. Sin. B 2017, 7, 3-17. [CrossRef] [PubMed]

31. Yoshikawa, Y.; Takano, O.; Kato, I.; Takahashi, Y.; Shima, F.; Kataoka, T. Ras inhibitors display an anti-metastatic effect by downregulation of lysyl oxidase through inhibition of the Ras-PI3K-Akt-HIF-1 $\alpha$ pathway. Cancer Lett. 2017, 410, 82-91. [CrossRef] [PubMed]

32. Krygowska, A.A.; Castellano, E. PI3K: A Crucial Piece in the RAS Signaling Puzzle. Cold Spring Harb. Perspect. Med. 2017. [CrossRef] [PubMed] 
33. Abubaker, J.; Bavi, P.; Al-Haqawi, W.; Jehan, Z.; Munkarah, A.; Uddin, S.; Al-Kuraya, K.S. PIK3CA alterations in Middle Eastern ovarian cancers. Mol. Cancer 2009, 8, 51. [CrossRef] [PubMed]

34. Cantley, L.C. The phosphoinositide 3-kinase pathway. Science 2002, 296, 1655-1657. [CrossRef] [PubMed]

35. Songyang, Z.; Shoelson, S.E.; Chaudhuri, M.; Gish, G.; Pawson, T.; Haser, W.G.; King, F.; Roberts, T.; Ratnofsky, S.; Lechleider, R.J.; et al. SH2 domains recognize specific phosphopeptide sequences. Cell 1993, 72, 767-778. [PubMed]

36. Yoakim, M.; Hou, W.; Songyang, Z.; Liu, Y.; Cantley, L.; Schaffhausen, B. Genetic analysis of a phosphatidylinositol 3-kinase SH2 domain reveals determinants of specificity. Mol. Cell. Biol. 1994, 14, 5929-5938. [CrossRef] [PubMed]

37. Gunther, U.L.; Weyrauch, B.; Zhang, X.; Schaffhausen, B. Nuclear magnetic resonance structure of the P395S mutant of the N-SH2 domain of the p85 subunit of PI3 kinase: An SH2 domain with altered specificity. Biochemistry 2003, 42, 11120-11127. [CrossRef] [PubMed]

38. Weber, T.; Schaffhausen, B.; Liu, Y.; Gunther, U.L. NMR structure of the N-SH2 of the p85 subunit of phosphoinositide 3-kinase complexed to a doubly phosphorylated peptide reveals a second phosphotyrosine binding site. Biochemistry 2000, 39, 15860-15869. [CrossRef] [PubMed]

39. Toulany, M.; Dittmann, K.; Kruger, M.; Baumann, M.; Rodemann, H.P. Radioresistance of K-Ras mutated human tumor cells is mediated through EGFR-dependent activation of PI3K-AKT pathway. Radiother. Oncol. 2005, 76, 143-150. [CrossRef] [PubMed]

40. Pi, J.; Jiang, J.; Cai, H.; Yang, F.; Jin, H.; Yang, P.; Cai, J.; Chen, Z.W. GE11 peptide conjugated selenium nanoparticles for EGFR targeted oridonin delivery to achieve enhanced anticancer efficacy by inhibiting EGFR-mediated PI3K/AKT and Ras/Raf/MEK/ERK pathways. Drug Deliv. 2017, 24, 1549-1564. [CrossRef] [PubMed]

41. Siempelkamp, B.D.; Rathinaswamy, M.K.; Jenkins, M.L.; Burke, J.E. Molecular mechanism of activation of class IA phosphoinositide 3-kinases (PI3Ks) by membrane-localized HRas. J. Biol. Chem. 2017, 292, 12256-12266. [CrossRef] [PubMed]

42. Bongiovanni, D.; Saccomani, V.; Piovan, E. Aberrant Signaling Pathways in T-Cell Acute Lymphoblastic Leukemia. Int. J. Mol. Sci. 2017, 18, 1904. [CrossRef] [PubMed]

43. Faes, S.; Dormond, O. PI3K and AKT: Unfaithful Partners in Cancer. Int. J. Mol. Sci. 2015, 16, $21138-21152$. [CrossRef] [PubMed]

44. Zi, D.; Zhou, Z.W.; Yang, Y.J.; Huang, L.; Zhou, Z.L.; He, S.M.; He, Z.X.; Zhou, S.F. Danusertib Induces Apoptosis, Cell Cycle Arrest, and Autophagy but Inhibits Epithelial to Mesenchymal Transition Involving PI3K/Akt/mTOR Signaling Pathway in Human Ovarian Cancer Cells. Int. J. Mol. Sci. 2015, 16, 27228-27251. [CrossRef] [PubMed]

45. Zhang, M.; Jang, H.; Gaponenko, V.; Nussinov, R. Phosphorylated Calmodulin Promotes PI3K Activation by Binding to the SH2 Domains. Biophys. J. 2017, 113, 1956-1967. [CrossRef] [PubMed]

46. Joyal, J.L.; Burks, D.J.; Pons, S.; Matter, W.F.; Vlahos, C.J.; White, M.F.; Sacks, D.B. Calmodulin activates phosphatidylinositol 3-kinase. J. Biol. Chem. 1997, 272, 28183-28186. [CrossRef] [PubMed]

47. Lu, S.; Jang, H.; Gu, S.; Zhang, J.; Nussinov, R. Drugging Ras GTPase: A comprehensive mechanistic and signaling structural view. Chem. Soc. Rev. 2016, 45, 4929-4952. [CrossRef] [PubMed]

48. Lu, S.; Jang, H.; Muratcioglu, S.; Gursoy, A.; Keskin, O.; Nussinov, R.; Zhang, J. Ras Conformational Ensembles, Allostery, and Signaling. Chem. Rev. 2016, 116, 6607-6665. [CrossRef] [PubMed]

49. Nussinov, R.; Muratcioglu, S.; Tsai, C.J.; Jang, H.; Gursoy, A.; Keskin, O. K-Ras4B/calmodulin/PI3K $\alpha$ : A promising new adenocarcinoma-specific drug target? Expert Opin. Ther. Targets 2016, 20, 831-842. [CrossRef] [PubMed]

50. Jang, H.; Banerjee, A.; Chavan, T.; Gaponenko, V.; Nussinov, R. Flexible-body motions of calmodulin and the farnesylated hypervariable region yield a high-affinity interaction enabling K-Ras4B membrane extraction. J. Biol. Chem. 2017, 292, 12544-12559. [CrossRef] [PubMed]

51. Krissinel, E.; Henrick, K. Inference of macromolecular assemblies from crystalline state. J. Mol. Biol. 2007, 372, 774-797. [CrossRef] [PubMed]

52. Miller, M.S.; Schmidt-Kittler, O.; Bolduc, D.M.; Brower, E.T.; Chaves-Moreira, D.; Allaire, M.; Kinzler, K.W.; Jennings, I.G.; Thompson, P.E.; Cole, P.A.; et al. Structural basis of nSH2 regulation and lipid binding in PI3K $\alpha$. Oncotarget 2014, 5, 5198-5208. [CrossRef] [PubMed] 
53. Bertacchini, J.; Heidari, N.; Mediani, L.; Capitani, S.; Shahjahani, M.; Ahmadzadeh, A.; Saki, N. Targeting PI3K/AKT/mTOR network for treatment of leukemia. Cell. Mol. Life Sci. 2015, 72, 2337-2347. [CrossRef] [PubMed]

54. Lu, S.; Jang, H.; Zhang, J.; Nussinov, R. Inhibitors of Ras-SOS Interactions. ChemMedChem 2016, 11, 814-821. [CrossRef] [PubMed]

55. Jiang, H.; Deng, R.; Yang, X.; Shang, J.; Lu, S.; Zhao, Y.; Song, K.; Liu, X.; Zhang, Q.; Chen, Y.; et al. Peptidomimetic inhibitors of APC-Asef interaction block colorectal cancer migration. Nat. Chem. Biol. 2017, 13, 994-1001. [CrossRef] [PubMed]

56. Ni, D.; Song, K.; Zhang, J.; Lu, S. Molecular Dynamics Simulations and Dynamic Network Analysis Reveal the Allosteric Unbinding of Monobody to H-Ras Triggered by R135K Mutation. Int. J. Mol. Sci. 2017, 18, 2249. [CrossRef] [PubMed]

57. Li, S.; Shen, Q.; Su, M.; Liu, X.; Lu, S.; Chen, Z.; Wang, R.; Zhang, J. Alloscore: A method for predicting allosteric ligand-protein interactions. Bioinformatics 2016, 32, 1574-1576. [CrossRef] [PubMed]

58. Lu, S.; Li, S.; Zhang, J. Harnessing allostery: A novel approach to drug discovery. Med. Res. Rev. 2014, 34, 1242-1285. [CrossRef] [PubMed]

59. Lu, S.; Zhang, J. Designed covalent allosteric modulators: An emerging paradigm in drug discovery. Drug Discov. Today 2017, 22, 447-453. [CrossRef] [PubMed]

60. Nolte, R.T.; Eck, M.J.; Schlessinger, J.; Shoelson, S.E.; Harrison, S.C. Crystal structure of the PI 3-kinase p85 amino-terminal SH2 domain and its phosphopeptide complexes. Nat. Struct. Biol. 1996, 3, 364-374. [CrossRef] [PubMed]

61. Pauptit, R.A.; Dennis, C.A.; Derbyshire, D.J.; Breeze, A.L.; Weston, S.A.; Rowsell, S.; Murshudov, G.N. NMR trial models: Experiences with the colicin immunity protein $\operatorname{Im} 7$ and the p $85 \alpha$ C-terminal SH2-peptide complex. Acta Crystallogr. Sect. D Biol. Crystallogr. 2001, 57, 1397-1404. [CrossRef]

62. Baspinar, A.; Cukuroglu, E.; Nussinov, R.; Keskin, O.; Gursoy, A. PRISM: A web server and repository for prediction of protein-protein interactions and modeling their 3D complexes. Nucleic Acids Res. 2014, 42, W285-W289. [CrossRef] [PubMed]

63. Keskin, O.; Nussinov, R.; Gursoy, A. PRISM: Protein-protein interaction prediction by structural matching. Methods Mol. Biol. 2008, 484, 505-521. [PubMed]

64. Ogmen, U.; Keskin, O.; Aytuna, A.S.; Nussinov, R.; Gursoy, A. PRISM: Protein interactions by structural matching. Nucleic Acids Res. 2005, 33, W331-W336. [CrossRef] [PubMed]

65. Mashiach, E.; Nussinov, R.; Wolfson, H.J. FiberDock: A web server for flexible induced-fit backbone refinement in molecular docking. Nucleic Acids Res. 2010, 38, W457-W461. [CrossRef] [PubMed]

66. Mashiach, E.; Nussinov, R.; Wolfson, H.J. FiberDock: Flexible induced-fit backbone refinement in molecular docking. Proteins 2010, 78, 1503-1519. [PubMed]

67. Meador, W.E.; Means, A.R.; Quiocho, F.A. Target enzyme recognition by calmodulin: 2.4 A structure of a calmodulin-peptide complex. Science 1992, 257, 1251-1255. [CrossRef] [PubMed]

68. Van Petegem, F.; Chatelain, F.C.; Minor, D.L., Jr. Insights into voltage-gated calcium channel regulation from the structure of the CaV1.2 IQ domain-Ca ${ }^{2+} /$ calmodulin complex. Nat. Struct. Mol. Biol. 2005, 12, 1108-1115. [CrossRef] [PubMed]

69. Shen, Y.; Guo, Q.; Zhukovskaya, N.L.; Drum, C.L.; Bohm, A.; Tang, W.J. Structure of anthrax edema factor-calmodulin-adenosine 5'-( $\alpha, \beta$-methylene)-triphosphate complex reveals an alternative mode of ATP binding to the catalytic site. Biochem. Biophys. Res. Commun. 2004, 317, 309-314. [CrossRef] [PubMed]

70. Case, D.A.; Cheatham, T.E., III; Darden, T.; Gohlke, H.; Luo, R.; Merz, K.M., Jr.; Onufriev, A.; Simmerling, C.; Wang, B.; Woods, R.J. The Amber biomolecular simulation programs. J. Comput. Chem. 2005, 26, 1668-1688. [CrossRef] [PubMed]

71. Duan, Y.; Wu, C.; Chowdhury, S.; Lee, M.C.; Xiong, G.; Zhang, W.; Yang, R.; Cieplak, P.; Luo, R.; Lee, T.; et al. A point-charge force field for molecular mechanics simulations of proteins based on condensed-phase quantum mechanical calculations. J. Comput. Chem. 2003, 24, 1999-2012. [CrossRef] [PubMed]

(C) 2018 by the authors. Licensee MDPI, Basel, Switzerland. This article is an open access article distributed under the terms and conditions of the Creative Commons Attribution (CC BY) license (http:/ / creativecommons.org/licenses/by/4.0/). 